\title{
Variáveis Associadas à Ansiedade Gestacional em Primigestas e Multigestas
}

\author{
Rafaela de Almeida Schiavo*,1, 2 \\ Orcid.org/0000-0001-7290-3610 \\ Olga Maria Piazentin Rolim Rodrigues ${ }^{3}$ \\ Orcid.org/0000-0002-5332-256X \\ Gimol Benzaquen Perosa ${ }^{4}$ \\ Orcid.org/0000-0002-7825-2537
}

\author{
${ }^{1}$ Universidade Paulista, Bauru, SP, Brasil \\ ${ }^{2}$ Instituto Municipal de Ensino Superior de São Manuel, São Manuel, SP, Brasil \\ ${ }^{3}$ Universidade Estadual Paulista “Júlio de Mesquita Filho”, Bauru, SP, Brasil \\ ${ }^{4}$ Universidade Estadual Paulista “Júlio de Mesquita Filho”, Botucatu, SP, Brasil
}

\section{Resumo}

Este estudo teve por objetivo descrever e comparar a ansiedade de primigestas e multigestas, no terceiro trimestre gestacional, bem como identificar, dentre as variáveis sociodemográficas e de gestação, as que se associaram para alta ansiedade. Participaram 479 gestantes que responderam a um instrumento de ansiedade (IDATE) e um questionário sobre variáveis sociodemográficas e da gestação. Compararam-se os grupos estatisticamente e montou-se um modelo de regressão logística para avaliar o peso das associações $(p<0,05)$. Os resultados indicaram que $36 \%$ das gestantes apresentaram sintomas de alta ansiedade no terceiro trimestre gestacional, com um predomínio das multigestas em relação às primigestas. Para as multigestas, a maior chance para alta ansiedade esteve associada à baixa renda e, para as primigestas, à ameaça de aborto no início da gestação. Tanto para primigestas como para multigestas, desejar o bebê apareceu como fator de proteção para alta ansiedade. A expressiva porcentagem de gestantes com sintomas de ansiedade justifica o oferecimento de serviços voltados à prevenção e promoção de saúde mental das gestantes, com atenção diferenciada às primigestas e multigestas.

Palavras-chave: Ansiedade, gravidez, primigesta, multigesta.

\section{Variables Associated with Gestational Anxiety in Primigravidas and Multigravidas}

\begin{abstract}
This study aimed to describe and compare anxiety on primigravidae and multiparous in the third quarter, as well as to identify, among sociodemographic variables and of pregnancy, which ones were associated

* Endereço para correspondência: Rua Quintino Bocaiúva, s/n, Aparecida de São Manuel, São Manuel, SP, Brasil 18650-000 Fone (fax) 2(14) 3841-3766. E-mail: professora.schiavo@gmail.com, olgarolim@fc.unesp. bregimol@fmb.unesp.br

Agradecemos a Fundação de Amparo à Pesquisa do Estado de São Paulo (FAPESP); Coordenação de Aperfeiçoamento de Pessoal de Nível Superior (CAPES) pelo auxílio financeiro para execução desse estudo.
\end{abstract}


with high anxiety. There were 479 pregnant women participants who responded to an anxiety instrument (STAI) and a questionnaire on sociodemographic variables and about pregnancy. The groups were statistically compared e a logistic regression model was composed to evaluate associations. The results indicated that $36 \%$ of pregnant women presented symptoms of high anxiety on third gestational trimester, with a predominance of the multiparous in relation to the primigravidae. For the multiparous, the highest chance for high anxiety was associated with low income and for the primigravidae to the threat of miscarriage in early pregnancy. For both primigravidae and multiparous, wanting the baby appeared as a protective factor for high anxiety. The expressive percentage of pregnant women with anxiety symptoms justifies the offering of prevention services and promotion of mental health for pregnant women, with a differentiated attention to primigravidae and multiparous.

Keywords: Anxiety, pregnant, primigravidae, multiparous.

\section{Las Variables Asociadas con la Ansiedad Gestacional en el Primigestas y Multigestas}

\section{Resumen}

Este estudio tuvo como objetivo describir y comparar la ansiedad en el primigestas y multigestas en tercer trimestre, y para identificar, entre las variables sociodemográficas y el embarazo, que se asocian a mayor ansiedad. Participaron en 479 mujeres embarazadas que respondieron a un instrumento de ansiedad (STAI) y un cuestionario sobre variables sociodemográficas y el embarazo. Los investigadores compararon los grupos estadísticamente y montaron un modelo de regresión logística para evaluar las asociaciones. Los resultados indicaron que el 36\% de las mujeres embarazadas tenían síntomas altos de ansiedad en tercer trimestre, con un predominio de multigestas en relación con el primigestas. Para multigestas la mayor oportunidad para alta ansiedad se asoció con bajas ingresos y para el primigestas con un aborto involuntario en el embarazo temprano. Ambos primigestas y multigestas quieren el bebé apareció como un factor protector para alta ansiedad. Un porcentaje significativo de mujeres embarazadas con síntomas de ansiedad justifica los servicios dirigidos a la prevención y promoción de la salud mental de las mujeres embarazadas, con una especial atención a las primigestas y multigestas.

Palabras clave: Ansiedad, embarazo, primigestas, multigestas.

A ansiedade faz parte do processo do desenvolvimento humano, podendo estar presente em todos os períodos do ciclo vital. É uma característica que acontece em momentos de medo, perigo ou tensão. Todavia, em alguns momentos da vida, a ansiedade é mais intensificada do que em outros. Dependendo do período, ela é considerada como um estado emocional esperado na mudança de emprego, frente ao resultado de um concurso ou exame médico, casamento, gravidez, nascimento de um filho, entre outros.

A ansiedade na gravidez é uma das alterações psicológicas mais comuns (Baptista, Baptista, \& Torre, 2006; Faisal-Cury, Araya, Zugaib, \& Menezes, 2010), variando de intensidade nas diferentes sociedades e culturas e seus índices são superiores aos do puerpério (Agrati et al., 2015; Baptista et al., 2006) tanto para pais como para mães (Figueiredo \& Conde, 2011). Devido à regulação negativa do $11 \beta$-HSD2 placentário, que aumenta a exposição fetal ao cortisol materno (O’Donnell et al., 2012), altos níveis de ansiedade na gestação podem resultar em complicações e intercorrências obstétricas, como o nascimento prematuro e baixo peso do bebê (Bener, 2013; Schetter \& Tanner, 2012).

A ansiedade gestacional está associada a menor tempo de aleitamento materno exclusivo (Adedinsewo, Fleming, Steiner, Meaney, \& Girard, 2014; Ystrom, 2012), podendo trazer, consequências negativas para a relação mãe/ bebê (Airosa \& Silva, 2013; Nardi, Rodrigues, 
Melchiori, Salgado, \& Tavano, 2015). Também pode incidir sobre o crescimento e causar atraso no desenvolvimento infantil (Beltrami, Moraes, \& Souza, 2013; Schetter \& Tanner, 2012; O'Donnell, Glover, Barker, \& O'Connor, 2014).

Quanto à saúde mental materna, a ansiedade gestacional associou-se com depressão gestacional (Schetter \& Tanner, 2012) e foi indicativo para ansiedade e depressão puerperal (Agrati et al., 2015; Coelho, Murray, Royal-Lawson, \& Cooper, 2011; Faisal-Cury et al., 2010) coincidindo com os estudos de Airosa e Silva (2013) e Nardi et al. (2015) que observaram que a ansiedade gestacional deixava a mulher vulnerável ao aparecimento de perturbações emocionais. Todavia, no estudo de Saviani-Zeoti e Petean (2015), os níveis elevados de ansiedade gestacional não apresentaram prejuízos na relação de apego com o feto.

Segundo a literatura, alguns dos fatores de risco para alta ansiedade gestacional são pouca idade materna, baixa escolaridade (Araújo, Pacheco, Pimenta, \& Kac, 2008), baixa renda (Simas, Souza, \& Scorsolini-Comin, 2013) e ameaça de abortamento (Alves, Alves, Ibiapina, Busgaib, \& Costa, 2010). Fonseca-Machado, Alves, Freitas, Monteiro e Gomes-Sponholz (2014), em revisão integrativa de literatura, identificaram que violência doméstica na gestação teve repercussão negativa para a saúde mental da grávida. $\mathrm{Na}$ revisão realizada para o presente artigo, não se encontrou referências a trabalhos que enfatizavam os fatores de proteção para alta ansiedade.

Poucos estudos abordaram a ansiedade gestacional em multigestas e primigestas e os resultados ainda são inconclusivos, ora associando a alta ansiedade a primigestas (Alves et al., 2007), ora a multigestas (Lopes, Vivian, Oliveira, Pereira, \& Piccinini, 2012), ou sem diferenças entre os dois grupos (Simas et al., 2013). Segundo Alves et al. (2007), a primigestação, ainda que seja um período de normalidade psíquica de caráter adaptativo é, também, um momento crítico marcado por angústias, pela eminência de ter de assumir um novo papel, que é ser mãe, que pode aumentar a ansiedade. Por questões culturais, frequentemente, essa nova responsabilidade é acompanhada de culpa e ansiedade, insegurança de não saber se irá corresponder integralmente ao ideal de maternagem difundido na sociedade ocidental, que espera mães, donas de casa e esposas perfeitas (Pitilin, Haracemiw, Marcon, \& Pelloso, 2013).

Para Simas et al. (2013), primigestas e multigestas são igualmente ansiosas, entretanto, apresentam ansiedade por diferentes motivos: as primigestas se angustiam mais pela inexperiência na tarefa de ser mãe enquanto que as multigestas são mais ambivalentes com a gravidez não planejada. Por outro lado, para Lopes et al. (2012), é um mito acreditar que a experiência da maternidade com o primogênito facilitaria a relação com o segundo filho. Segundo os autores, as multigestas, especialmente aquelas que estão em situação de vulnerabilidade econômica e têm que assumir inúmeros encargos, vivem uma violência social silenciada, onde não há espaço para erros, raiva e tristeza, o que pode justificar altos índices de ansiedade.

No puerpério, os resultados também são discrepantes. Se Faisal-Cury e Menezes (2006), no Brasil, identificaram que multíparas apresentavam maiores índices de ansiedade que primíparas, na pesquisa de Veríssimo (2010), em Portugal, constatou-se que as primíparas, quando comparadas com as multíparas, possuíam maior ansiedade.

Além da literatura incipiente e dos dados inconclusivos a respeito dos possíveis problemas de saúde mental em primigestas e multigestas, algumas pesquisas têm se interessado com a repercussão que o número de filhos pode trazer para a qualidade de vida do casal assim como a sua relação com as crianças (Camarneiro \& Justo, 2012; Pereira, Fonseca, Oliveira, Souza, \& Mello, 2013; Saviani-Zeoti \& Petean, 2015). Há dados indicando que primigestas têm maior satisfação conjugal, preocupação com o feto e melhor percepção de sua qualidade de vida que multigestas (Camarneiro \& Justo, 2012). Por outro lado, no estudo de Pereira et al. (2013), uma porcentagem maior de multíparas amamentaram na primeira hora após o nascimento do que as primíparas. 
Como se pode perceber, as pesquisas que comparam os níveis de ansiedade entre primigestas e multigestas, assim como seus fatores associados, ainda são escassas com diferentes resultados, possivelmente devido a amostras, instrumentos de avaliação e contextos de investigação diversos. Tais constatações sugerem a necessidade de maiores investigações a respeito, com o intuito de auxiliar no oferecimento de serviços de apoio diferenciados a gestantes primigestas e multigestas, focados no controle da ansiedade materna neste período do ciclo vital. Fundamentado nas considerações acima, este estudo teve por objetivo descrever e comparar a ansiedade de primigestas e multigestas, no terceiro trimestre gestacional, bem como identificar, dentre as variáveis sociodemográficas e de gestação, as que se associaram para alta ansiedade.

\section{Método}

Trata-se de um estudo transversal, que avaliou características sociodemográficas, de percepção da maternidade e a ansiedade de gestantes. Para compor a amostra, foram escolhidos locais que concentravam grande número de atendimentos à gestante pelo Sistema Único de Saúde (SUS), em três cidades de médio porte do interior paulista.

\section{Participantes}

Entre 2010 e 2015, foram convidadas a participar da pesquisa 485 gestantes no terceiro trimestre que aguardavam atendimento pré-natal em Unidades Básicas de Saúde (UBS). Destas, 479 aceitaram participar da pesquisa e, enquanto aguardavam respondiam os instrumentos, individualmente, em uma sala apropriada. Participaram 222 primigestas e 257 multigestas.

\section{Instrumentos}

Utilizou-se um formulário único elaborado pelos autores deste estudo, com 11 questões, sendo cinco questões referentes aos dados sociodemográficos (idade, estado civil, escolaridade, renda familiar, número de filhos), três questões referentes aos antecedentes clínicos (saúde e saúde mental anterior à gestação, saúde durante a gestação) e duas questões referentes à gestação (ameaça de aborto da gestação atual e abortamento anterior). Foi também perguntado às gestantes se ela e o parceiro haviam desejado (ou não) o bebê.

Para a avaliação da ansiedade utilizou-se o Inventário de Ansiedade Traço/Estado (IDATE), elaborado por Spielberger, Gorsuch e Lushene (1970), traduzido e adaptado para o português por Biaggio e Natalício (1979/2003). É um instrumento de autoaplicação, para jovens e adultos, composto de duas escalas que avaliam Ansiedade estado (A-Estado) e Ansiedade traço (A-Traço). A partir de 20 itens, o indivíduo deve avaliar a si mesmo, a partir de uma escala Likert de quatro pontos.

Em relação à suas qualidades psicométricas, sua fidedignidade no teste-reteste foi de 0,74 para Ansiedade estado e 0,83 para Ansiedade traço. A consistência interna, avaliada pelo Alfa de Cronbach, foi de 0,88 em relação à Ansiedade estado feminino enquanto que para a Ansiedade traço feminino foi de 0,87 (Biaggio \& Natalício, 1979/2003). Para o presente estudo, calculou-se a consistência interna pelo Alfa de Cronbach para ansiedade estado e traço de gestantes no terceiro trimestre. Obteve-se um alfa de 0.67 para ansiedade estado e 0.63 para ansiedade traço, indicando que os índices de consistência para a versão em português do IDATE aplicado em gestantes, foram de moderado a bom. Como indicativo de sintomas de alta ansiedade foi utilizado o ponto de corte de escore igual ou acima do percentil 75 , já utilizado em outros estudos (Perosa, Canavez, Silveira, Padovani, \& Peraçou, 2009; Perosa, Silveira, \& Canavez, 2008).

\section{Procedimento de Coleta de Dados}

O recrutamento das participantes ocorreu nas próprias UBS das três cidades do interior paulista. A pesquisadora, primeira autora deste estudo, se dirigia a todas as gestantes que estavam no terceiro trimestre e que aguardavam na sala de espera para serem atendidas pelo obstetra 
e fazia o convite para participação da pesquisa, informando que deveriam responder a dois instrumentos, e que um deles avaliaria o nível de ansiedade. As gestantes que aceitaram participar, após assinarem o Termo de Consentimento Livre e Esclarecido, em ambiente reservado, na unidade de saúde, responderam ao Formulário e o IDATE. Para gestantes não alfabetizadas e para aquelas que, por algum motivo, preferiam não ler, a pesquisadora lia as questões do inventário e preenchia a folha de resposta referente a esse instrumento. Todas as gestantes eram informadas sobre o resultado do IDATE logo após a sua aplicação, as que pontuavam para alta ansiedade foram orientadas a procurar por atendimento psicológico clínico especializado, a pesquisadora entregava para estas gestantes o telefone e endereço do local para busca desse atendimento.

\section{Procedimento de Análise de Dados}

As variáveis sociodemográficas, de antecedentes clínicos e gestacionais, numéricas e categóricas, foram binarizadas e organizadas na base de dados do programa Statistical Package for the Social Sciences (SPSS, versão 21.0; Chicago INc II, USA), para posterior análise dos dados. O critério para binarizar a idade foi o proposto pela Organização Mundial da Saúde (World Health Organization [WHO], 1986) que define como adolescência as idades entre 10 e 19 anos. O IDATE foi corrigido segundo as normas específicas do manual e os resultados binarizados, considerando o percentil 75 como um marcador de alta ansiedade. As variáveis numéricas (idade materna e número de filhos) e as categóricas (todas do tipo dummy) foram analisadas por meio de estatística descritiva (frequência e porcentagem). Antes de proceder à análise estatística inferencial, foi aplicado o teste de Shapiro Wilk, para testar a normalidade das variáveis. Para comparar as primigestas e multigestas quanto às variáveis sociodemográficas, relacionadas aos antecedentes clínicos e gestacionais e à alta ansiedade, utilizou-se o teste de qui-quadrado. Para identificar possíveis variáveis preditoras de risco e proteção para alta ansiedade, foi aplicado um modelo de regressão logística com todas as va- riáveis dicotômicas em bloco único, para o total da amostra, e, separadamente, para as primigestas e multigestas. O nível de significância adotado foi de $p<0,05$.

\section{Procedimentos Éticos}

O projeto foi aprovado pelo Comitê de Ética em Pesquisa da Faculdade de Medicina de Botucatu Universidade Estadual Paulista «Júlio de Mesquita Filho» - UNESP (processo ${ }^{\circ} 4166$ 2012) e pela Faculdade de Ciências da UNESP campus de Bauru (processo no 614/46/01/09). A coleta dos dados foi autorizada pelos responsáveis dos três centros que atendiam as grávidas na atenção básica. A coleta de dados só se deu após assinatura do termo de Consentimento Livre e Esclarecido que foi entregue às participantes. Todas as 479 participantes, após responderem aos instrumentos, aguardavam a correção da pesquisadora no local de aplicação, a qual imediatamente fornecia o resultado. Aquelas que pontuaram para alta ansiedade foram encaminhadas para locais de atendimento profissional especializado em sua cidade.

\section{Resultados}

Em primeiro lugar, serão apresentados os dados sociodemográficos e referentes à maternidade. A seguir, as características de ansiedade traço e estado no terceiro trimestre de gestação entre primigestas e multigestas, os fatores associados com alta ansiedade no terceiro trimestre e os fatores associados com alta ansiedade em primigistas e multigestas.

Pela leitura da Tabela 1 é possível verificar que as multigestas eram significantemente mais velhas $(p<0,001)$, tinham em média 27 anos $(D P=5,4)$ e as primigestas tinham idade média de 20 anos $(D P=5,5)$. Quanto ao número de filhos, 36,6\% das multigestas já tinham um filho, $34,4 \%$ tinham dois e $42,2 \%$, três ou mais filhos. As multigestas, também, tinham menor escolaridade $(p=0,009)$ e uma porcentagem maior de mães $(p=0,004)$ e de pais $(p=0,049)$ que não desejaram o bebê, quando comparadas com as primigestas. 
Tabela 1

Comparação entre Primigestas e Multigestas segundo as Características Sociodemográficas e Referentes à Maternidade $(N=479)$

\begin{tabular}{|c|c|c|c|c|c|c|c|}
\hline \multirow{3}{*}{ Variáveis associadas } & \multicolumn{4}{|c|}{ Paridade } & \multirow[b]{3}{*}{ Total } & \multirow{3}{*}{$X^{2}$} & \multirow{3}{*}{$p-$ valor* } \\
\hline & \multicolumn{2}{|c|}{$\begin{array}{c}\text { Primigesta } \\
n=222\end{array}$} & \multicolumn{2}{|c|}{$\begin{array}{l}\text { Multigesta } \\
n=257\end{array}$} & & & \\
\hline & $F$ & $\%$ & $F$ & $\%$ & & & \\
\hline Idade & & & & & & 107,395 & $<0,001$ \\
\hline$\leq 19$ anos & 101 & 88,6 & 13 & 11,4 & $114(24 \%)$ & & \\
\hline$\geq 20$ anos & 121 & 33,2 & 244 & 66,8 & $365(76 \%)$ & & \\
\hline Estado Conjugal & & & & & & 2,674 & 0,102 \\
\hline Vive com parceiro(a) & 164 & 44,3 & 206 & 55,7 & $370(77 \%)$ & & \\
\hline Não vive com parceiro(a) & 58 & 53,2 & 51 & 46,8 & $109(23 \%)$ & & \\
\hline Escolaridade & & & & & & 6,479 & 0,011 \\
\hline$\leq$ nove anos & 84 & 39,8 & 127 & 60,2 & $211(44 \%)$ & & \\
\hline$>$ nove anos & 138 & 51,5 & 130 & 48,5 & $268(56 \%)$ & & \\
\hline Ocupação Remunerada & & & & & & 0,018 & 0,893 \\
\hline Sim & 73 & 45,9 & 86 & 54,1 & $159(33 \%)$ & & \\
\hline Não & 149 & 46,6 & 171 & 53,4 & $320(67 \%)$ & & \\
\hline Renda Familiar & & & & & & 0,628 & 0,428 \\
\hline$\leq 2$ salários mínimos & 206 & 45,9 & 243 & 54,1 & $449(94 \%)$ & & \\
\hline$>2$ salários mínimos & 16 & 53,3 & 14 & 46,7 & $30(6.3 \%)$ & & \\
\hline Saúde anterior à gestação & & & & & & 2,737 & 0,098 \\
\hline Normal & 206 & 47,6 & 227 & 52,4 & $433(90 \%)$ & & \\
\hline Algum problema & 16 & 34,8 & 30 & 65,2 & $46(10 \%)$ & & \\
\hline Saúde durante a gestação & & & & & & 1,482 & 0,223 \\
\hline Normal & 189 & 47,6 & 208 & 52,4 & $397(83 \%)$ & & \\
\hline Algum problema & 33 & 40,2 & 49 & 59,8 & $82(17 \%)$ & & \\
\hline Ameaça de aborto & & & & & & 0,267 & 0,605 \\
\hline Sim & 21 & 42,9 & 28 & 57,1 & $49(10 \%)$ & & \\
\hline Não & 201 & 46,7 & 229 & 53,3 & $430(90 \%)$ & & \\
\hline Desejou o bebê & & & & & & 8,223 & 0,004 \\
\hline Sim & 147 & 51,8 & 137 & 48,2 & $284(59 \%)$ & & \\
\hline Não & 75 & 38,5 & 120 & 61,5 & $195(41 \%)$ & & \\
\hline Parceiro desejou o bebê & & & & & & 3,892 & 0,049 \\
\hline Sim & 158 & 49,5 & 161 & 50,5 & $319(67 \%)$ & & \\
\hline Não & 64 & 40,0 & 96 & 60,0 & $160(33 \%)$ & & \\
\hline
\end{tabular}

Nota. F - frequência; \% - porcentagem; $\mathrm{X}^{2}$ - Qui-Quadrado.

* $p$-valor para o teste do Qui-Quadrado. 
Tabela 2

Diferença entre Primigestas e Multigestas quanto à Alta Ansiedade Traço/Estado $(N=479)$

\begin{tabular}{|c|c|c|c|c|c|c|c|c|}
\hline & \multicolumn{4}{|c|}{ Paridade } & & & \multirow{3}{*}{$\mathrm{X}^{2}$} & \multirow{3}{*}{$p$ - valor* } \\
\hline & \multicolumn{2}{|c|}{ Primigesta } & \multicolumn{2}{|c|}{ Multigesta } & \multicolumn{2}{|c|}{ Total } & & \\
\hline & $F$ & $\%$ & $F$ & $\%$ & $F$ & $\%$ & & \\
\hline Ansiedade Traço & & & & & & & 7,316 & 0,007 \\
\hline Sem alta ansiedade & 156 & 51,0 & 150 & 49,0 & 306 & 63,9 & & \\
\hline Com alta ansiedade & 66 & 38,2 & 107 & 61,8 & 173 & 36,1 & & \\
\hline Total & 222 & 46,3 & 257 & 53,7 & 479 & 100 & & \\
\hline Ansiedade Estado & & & & & & & 5,802 & 0,016 \\
\hline Sem alta ansiedade & 154 & 50,5 & 151 & 49,5 & 305 & 63,7 & & \\
\hline Com alta ansiedade & 68 & 39,1 & 106 & 60,9 & 174 & 36,3 & & \\
\hline Total & 222 & 46,3 & 257 & 53,7 & 479 & 100 & & \\
\hline
\end{tabular}

Nota. F - frequência; \% - porcentagem; $\mathrm{X}^{2}$ - Qui-Quadrado.

*p-valor para o teste do Qui-Quadrado.

Considerando a amostra total, traço ou estado, cerca de $36 \%$ das gestantes apresentaram alta ansiedade. Na Tabela 2 pode se observar, tam- bém, que, de forma significativa uma porcentagem maior de multigestas apresentou alta ansiedade, tanto traço $(61,8 \%)$ quanto estado $(60,9 \%)$ em relação às primigestas.

Tabela 3

Fatores Associados para Maior e Menor Chance de Alta Ansiedade Traço/Estado, no Terceiro Trimestre de Gestação $(N=479)$

\begin{tabular}{lcccccc}
\hline & \multicolumn{3}{c}{ Ansiedade Traço } & \multicolumn{3}{c}{ Ansiedade Estado } \\
\cline { 2 - 7 } & $p$-valor* & OR & IC 95\% & $p$-valor* & OR & IC 95\% \\
\hline Idade & 0,490 & 1,185 & 0,$732 ; 1,921$ & 0,068 & 1,593 & 0,$966 ; 2,628$ \\
Estado conjugal & 0,777 & 1,071 & 0,$667 ; 1,719$ & 0,766 & 1,079 & 0,$665 ; 1,742$ \\
Escolaridade & 0,109 & 1,395 & 0,$929 ; 2,093$ & 0,317 & 1,234 & 0,$818 ; 1,863$ \\
Ocupação remunerada & 0,220 & 0,757 & 0,$485 ; 1,181$ & 0,629 & 0,896 & 0,$574 ; 1,398$ \\
Renda familiar & 0,247 & 1,736 & 0,$682 ; 4,419$ & 0,082 & 2,335 & 0,$898 ; 6,069$ \\
Saúde anterior à gestação & 0,266 & 1,464 & 0,$748 ; 2,865$ & 0,135 & 1,672 & 0,$852 ; 3,280$ \\
Saúde durante a gestação & 0,520 & 1,187 & 0,$704 ; 2,001$ & 0,679 & 0,893 & 0,$521 ; 1,529$ \\
Ameaça de aborto & 0,920 & 1,034 & 0,$539 ; 1,983$ & 0,021 & 2,126 & 1,$122 ; 4,027$ \\
Histórico de abortamento & 0,066 & 1,589 & 0,$970 ; 2,600$ & 0,029 & 1,734 & 1,$057 ; 2,845$ \\
Desejar o bebê & 0,031 & 0,571 & 0,$343 ; 0,949$ & 0,003 & 0,455 & 0,$273 ; 0,758$ \\
Parceiro desejar o bebê & 0,037 & 0,568 & 0,$334 ; 0,967$ & 0,122 & 0,655 & 0,$384 ; 1,120$ \\
\hline
\end{tabular}

Nota. OR - Odds Ratio; IC - Intervalo de Confiança.

* $p$-valor para o teste de Regressão Logística. 
$\mathrm{Na}$ análise de predição para o total da amostra, pode se observar que nenhuma variável sociodemográfica mostrou associação significativa com alta ansiedade traço e estado. Todavia, verificou-se que a mãe ter desejado o bebê, assim como seu parceiro, diminuiu a chance de apresentar alta ansiedade traço no terceiro trimestre de gestação. Da mesma forma, a mãe ter desejado o bebê diminuiu a chan- ce de pontuar para alta ansiedade estado, no terceiro trimestre de gestação. Gestantes que tiveram histórico clínico de ameaça de aborto no início da gravidez e abortamento anterior tiveram maior chance de apresentar alta ansiedade estado (Tabela 3).

A seguir, serão apresentados os fatores associados à alta ansiedade traço e estado em primigestas (Tabela 4) e em multigestas (Tabela 5), no terceiro trimestre de gestação.

Tabela 4

Fatores Associados para Maior e Menor Chance de Alta Ansiedade Traço/Estado em Primigestas no Terceiro Trimestre de Gestação $(n=222)$

\begin{tabular}{|c|c|c|c|c|c|c|}
\hline & \multicolumn{6}{|c|}{ Primigestas } \\
\hline & \multicolumn{3}{|c|}{ Ansiedade Traço } & \multicolumn{3}{|c|}{ Ansiedade Estado } \\
\hline & $p$-valor* & OR & IC $95 \%$ & $p$-valor* & OR & IC 95\% \\
\hline Idade & 0,933 & 0,970 & 0,$481 ; 1,958$ & 0,514 & 1,273 & 0,$617 ; 2,626$ \\
\hline Estado conjugal & 0,504 & 0,779 & 0,$374 ; 1,622$ & 0,567 & 1,235 & 0,$600 ; 2,543$ \\
\hline Escolaridade & 0,734 & 1,129 & 0,$569 ; 2,226$ & 0,789 & 1,100 & 0,$549 ; 2,204$ \\
\hline Ocupação Remunerada & 0,192 & 0,602 & 0,$281 ; 1,290$ & 0,942 & 0,973 & 0,$462 ; 2,047$ \\
\hline Renda Familiar & 0,355 & 0,554 & 0,$158 ; 1,938$ & 0,757 & 0,816 & 0,$226 ; 2,947$ \\
\hline Saúde anterior à gestação & 0,923 & 1,061 & 0,$323 ; 3,480$ & 0,703 & 1,253 & 0,$393 ; 3,996$ \\
\hline Saúde durante a gestação & 0,412 & 0,678 & 0,$268 ; 1,716$ & 0,381 & 0,662 & 0,$263 ; 1,666$ \\
\hline Ameaça de aborto & 0,136 & 2,164 & 0,$785 ; 5,964$ & 0,017 & 3,464 & 1,$245 ; 9,640$ \\
\hline Histórico de abortamento & 0,196 & 1,842 & 0,$730 ; 4,651$ & 0,086 & 2,263 & 0,$891 ; 5,749$ \\
\hline Desejo pelo bebê & 0,839 & 0,910 & 0,$365 ; 2,267$ & 0,033 & 0,390 & 0,$164 ; 0,928$ \\
\hline Parceiro desejou bebê & 0,020 & 0,322 & 0,$124 ; 0,837$ & 0,358 & 0,653 & 0,$263 ; 1,620$ \\
\hline
\end{tabular}

Nota. OR - Odds Ratio; IC - Intervalo de Confiança. *p-valor para o teste de Regressão Logística.

$\mathrm{Na}$ Tabela 4 , pode se perceber que para as primigestas, desejar o bebê, apareceu como fator de proteção, enquanto que ameaça de aborto, aumentou em 3,464 a chance para alta ansiedade estado. Quanto à ansiedade traço, o parceiro desejar o bebê diminuiu a chance de a gestante apresentar alta ansiedade.

Com relação às multigestas, a baixa renda aumentou a chance de ter alta ansiedade traço e estado enquanto ter desejado o bebê diminuiu a chance, tanto para um quanto para outro tipo de ansiedade (Tabela 5).

\section{Discussão}

As características sociodemográficas das participantes se assemelharam aos de outros estudos brasileiros que avaliaram gestantes e puérperas (Beltrami et al., 2013; Faisal-Cury \& Menezes, 2006; Morais, Lucci \& Otta, 2013), 
Tabela 5

Fatores Associados para Maior e Menor Chance de Alta Ansiedade Traço/Estado em Multigestas no Terceiro Trimestre de Gestação $(n=257)$

Multigesta

\begin{tabular}{lcccccc}
\cline { 2 - 7 } & \multicolumn{3}{c}{ Ansiedade Traço } & \multicolumn{3}{c}{ Ansiedade Estado } \\
\cline { 2 - 7 } & $p$-valor* & OR & IC 95\% & $p$-valor* & OR & IC 95\% \\
\hline Idade & 0,802 & 0,856 & 0,$253 ; 2,892$ & 0,137 & 2,641 & 0,$735 ; 9,489$ \\
Estado conjugal & 0,328 & 1,405 & 0,$711 ; 2,773$ & 0,758 & 0,898 & 0,$455 ; 1,775$ \\
Escolaridade & 0,119 & 1,553 & 0,$894 ; 2,698$ & 0,276 & 1,355 & 0,$785 ; 2,340$ \\
Ocupação remunerada & 0,761 & 0,914 & 0,$512 ; 1,632$ & 0,655 & 0,878 & 0,$495 ; 1,557$ \\
Renda familiar & 0,043 & 5,094 & 1,$050 ; 24,704$ & 0,021 & 6,622 & 1,$337 ; 32,793$ \\
Saúde anterior à gestação & 0,236 & 1,702 & 0,$706 ; 4,104$ & 0,167 & 1,836 & 0,$775 ; 4,352$ \\
Saúde durante a gestação & 0,129 & 1,727 & 0,$853 ; 3,496$ & 0,793 & 1,098 & 0,$545 ; 2,215$ \\
Ameaça de aborto & 0,146 & 0,503 & 0,$199 ; 1,270$ & 0,360 & 1,493 & 0,$633 ; 3,525$ \\
Histórico de abortamento & 0,368 & 1,327 & 0,$717 ; 2,453$ & 0,162 & 1,538 & 0,$841 ; 2,813$ \\
Desejo pelo bebê & 0,007 & 0,397 & 0,$203 ; 0,776$ & 0,015 & 0,439 & 0,$226 ; 0,850$ \\
Parceiro desejou bebê & 0,641 & 0,848 & 0,$425 ; 1,693$ & 0,303 & 0,698 & 0,$352 ; 1,384$ \\
\hline
\end{tabular}

Nota. OR - Odds Ratio; IC - Intervalo de Confiança.

* p-valor para o teste de Regressão Logística.

o que permite comparações entre o presente estudo e os demais. Como em outras pesquisas (Borges, Santos, Nascimento, Chofakian, \& Gomes-Sponholz, 2016; Conceição \& Fernandes, 2015) houve um elevado número de gestantes que relatou não ter desejado a gravidez (41\%), especialmente as multigestas, sugerindo que, apesar de constar em lei a garantia de planejamento familiar como uma responsabilidade do Estado, com o objetivo de proporcionar condições para que homens e mulheres possam ter acesso a informações sobre os meios contraceptivos (Prates, Abib, \& Oliveira, 2008), ela pode não estar ocorrendo de forma efetiva. Para alguns autores, isso se deve à limitação e deficiência na infraestrutura da assistência, a pouca informação das mulheres sobre contracepção ou à resistência do cônjuge pela procura dos serviços de planejamento familiar (Caetano, 2014; Penaforte et al., 2010; Pitilin et al., 2013).
As multigestas eram mais velhas do que as primigestas. Esperava-se que pelo fato das multigestas serem mais velhas, indicativo de maior experiência, conhecimento e capacidade para antecipar ocorrências adquiridas na educação dos outros filhos, elas apresentariam baixa ansiedade em relação às primigestas. Entretanto, neste estudo, como no de Lopes et al. (2012) um maior número de multigestas apresentou alta ansiedade, talvez associada à sua baixa renda e número de filhos, como será discutido mais adiante.

Este estudo permitiu identificar que cerca de $36 \%$ das participantes, no último trimestre da gestação, apresentaram alta ansiedade traço e ansiedade estado. Trata-se de proporção inferior à de outros estudos nacionais, provavelmente, devido ao ponto de corte adotado para alta ansiedade neste trabalho ser superior ao dos outros estudos (Baptista et al., 2006; Faisal-Cury \& Menezes, 2006). 
As multigestas também tinham menor escolaridade que as primigestas, possivelmente, porque as multigestas não foram favorecidas pelas novas políticas educacionais de progressão continuada, iniciadas no ano de 1996, responsáveis por níveis mais altos de escolaridade de gestantes e puérperas, em pesquisas recentes (Beltrami et al., 2013; Faisal-Cury \& Menezes, 2006; Morais et al., 2013). Estudos têm apontado que a multiparidade está associada a menor grau de instrução materna que, por sua vez, se reflete em menores salários, menor renda familiar e maior vulnerabilidade econômica (Moura, Silva, Gomes, Almeida, \& Evangelista, 2010; Prates et al., 2008; Severinski, Mamula, Severinski, \& Mamula, 2009).

Confirmando as colocações de Simas et al. (2013), a porcentagem de gestantes com alta ansiedade, assim como os fatores de risco sociodemográficos, clínicos e gestacionais, diferiram nos dois grupos: se para as multigestas a baixa renda apareceu como fator de risco para alta ansiedade (traço e estado), para as primigestas foi à ameaça de aborto no início da gravidez (estado).

Na medida em que $42,2 \%$ das multigestas tinham mais de três filhos, a vinda de mais um filho, especialmente para as multigestas de baixa renda, pode ter levado a antever uma nova reconfiguração do sistema familiar, com mais responsabilidades e possíveis prejuízos em sua perspectiva de futuro, dificuldades financeiras e de qualidade de vida, gerando incertezas e ansiedade (Borges, Ferreira, Mariutti, \& Almeida, 2011; Prates et al., 2008; Simas et al., 2013). No estudo de Pitilin et al. (2013), com multíparas de quatro ou mais filhos, usuárias do SUS, observou-se que a vulnerabilidade econômica não se limitava à privação de renda mas, também, interferia na possibilidade de obter trabalho com qualidade e remuneração adequada, no acesso a serviços públicos e na dinâmica da vida familiar.

Se as dificuldades financeiras podem ter aumentado a tensão frente ao nascimento de um novo filho em multigestas, a possibilidade de perda fetal aumentou a chance de alta ansiedade estado nas primigestas. Muitas vezes, a ameaça de aborto é um quadro com fisiopatologia desco- nhecida, que exige diversos tipos de intervenção que, se por um lado, procuram evitar a perda fetal, por outro aumentam a sensação de imprevisibilidade e decorrente ansiedade (Alves et al., 2010).

Tanto em primigestas como multigestas, o histórico de abortamento aumentou significativamente a chance para alta ansiedade (estado), possivelmente porque essa experiência faz com que as gestantes vivenciem a nova gravidez com sentimentos ambivalentes de apego com relação ao bebê, ao lado de insegurança e medo (Freire $\&$ Chatelard, 2009). No estudo de Fernandes et al. (2012), tanto mulheres que abortaram espontaneamente como as que o provocaram aborto, apresentaram em nova gravidez, ansiedade associada ao temor de um novo aborto.

Se a possibilidade de perda da criança apareceu como fator de risco, desejar o filho se associou a menor chance de primigestas e multigestas apresentarem sintomas de alta ansiedade (traço e estado). Para Pitilin et al. (2013), na grande maioria das vezes, mesmo se não há planejamento da gravidez e as mulheres acreditam ter engravidado em um momento errado, à angustia e ansiedade inicial, advêm sentimentos de resignação, adaptação e, no decorrer da gestação, elas passam a desejar o bebê. Em alguns estudos, mulheres que não planejaram a gravidez por dificuldades financeiras e conjugais têm a crença de que o bebê vem para renovar o relacionamento familiar, inclusive o conjugal, e o desejo pelo filho passa a ser uma experiência satisfatória, até mesmo salvadora, elevando sua autoestima e protegendo-as de problemas emocionais (Moura et al., 2010; Prietsch, González-Chica, Cesar, \& Mendoza-Sassi, 2011; Simas et al., 2013).

O parceiro desejar o bebê diminuiu as chances para alta ansiedade traço em primigestas, mostrando que o desejo por um filho e o possível envolvimento paterno durante a gestação podem ter atenuado as incertezas quanto aos cuidados, especialmente das primíparas. Segundo Simas et al. (2013), quando o parceiro aceita a gravidez e deseja ser pai, ele se envolve mais no processo, assume com a gestante os preparativos para a chegada do bebê, oferece apoio emocional, procura fazer contato com o bebê, bem como dá 
ouvidos às preocupações e anseios da mulher, constituindo-se em importante apoio social.

O presente estudo contribuiu com avanços nesta área de pesquisa, pois comparou a ansiedade de primigestas e multigestas, no terceiro trimestre gestacional, e identificou, dentre as variáveis sociodemográficas e de gestação, as que se associaram para alta ansiedade em cada grupo, permitindo que medidas socioeducativas possam ser elaboradas a partir dos dados identificados como maior atenção à saúde emocional de gestantes, principalmente multigestas de baixa renda e primigestas que passaram por ameaça de aborto no início da gravidez.

\section{Considerações Finais}

Os resultados obtidos trazem avanços ao conhecimento na área, contudo, é importante considerar algumas limitações deste estudo que podem ajudar no planejamento de novas investigações. A primeira limitação se refere ao instrumento usado para medir ansiedade, o IDATE, que também foi utilizado em outros estudos para identificar níveis de ansiedade ao final da gestação (Agrati et al., 2015; Araújo et al., 2008; Baptista et al., 2006; O'Donnell et al., 2012; Roos, Faure, Louchner, Vythilingum, \& Stein, 2013). Apesar da confiabilidade, calculada para este estudo, a partir do Alfa de Cronbach, ter mostrado índices de moderado a bom, o IDATE não é um instrumento específico para grávidas, pois alguns sintomas avaliados como ansiedade podem ser características esperadas em uma gravidez. Recomenda-se que novas investigações ocorram, no sentido de identificar qual o melhor instrumento para medir ansiedade em gestantes, ou se elabore um instrumento específico para esta população.

Outra possível limitação do estudo pode ter sido o fato de se ter binarizado todas as variáveis, inclusive as contínuas, como a idade e renda, e que possivelmente, se fossem consideradas em sua forma continua possibilitaria uma análise mais acurada da ansiedade nas diferentes faixas etárias e socioeconômicas, na regressão logística. Também, o fato da amostra não ser probabilística, mas de conveniência, limita a ge- neralização dos resultados. Recomenda-se que, em novas investigações, além de não binarizar as variáveis, se sorteiem os sujeitos, aumentando a generalidade dos resultados.

Apesar das limitações, o presente estudo, identificou alta ansiedade em $36 \%$ de gestantes usuárias do SUS, no terceiro trimestre gestacional, principalmente entre as multigestas. A associação já comprovada da ansiedade gestacional com complicações obstétricas, com depressão puerperal, além dos prejuízos que pode acarretar ao crescimento e desenvolvimento infantil, alerta para a necessidade do profissional que atende no pré-natal dar uma atenção especial à saúde emocional da gestante.

O estudo reforça também que os fatores de risco à alta ansiedade são diferentes em primigestas e multigestas, justificando o oferecimento de uma atenção diferenciada às grávidas pela primeira vez e às que já passaram por gestações anteriores. No caso das primigestas, ter vivenciado uma ameaça de abortamento no início da gestação, apareceu como a principal variável preditora de alta ansiedade no terceiro trimestre, o que sinaliza para a importância de oferecer o máximo de informações, aliado a suporte psicológico, naquele momento. Frequentemente, não se encontram causas que justifiquem a ameaça de aborto (Alves et al., 2010) o que leva muitas mulheres a percebê-la como falha individual, associada a sentimentos de culpa e fantasias de "merecido castigo", aspectos relevantes a serem considerados nas ações de atenção psicológica em saúde.

Os sentimentos e fantasias das primigestas precisam ser abordados desde o momento em que ocorreu a ameaça de aborto para prevenir a cronificação do estado emocional da gestante que, em última análise, pode influenciar negativamente, tanto a saúde mental materna e o parto, como a saúde física e emocional do bebê. Entretanto, ainda são necessárias pesquisas para verificar que tipo de atendimento, individual ou em grupo, pode prevenir de forma mais eficiente o agravamento da ansiedade no final da gestação.

Com relação às multigestas, uma alta porcentagem não desejava o bebê $(60 \%)$ e a alta ansiedade associou-se com baixa renda, uma 
variável estrutural em que os profissionais da saúde pouco podem atuar, sendo, portanto, relevantes políticas públicas nos atendimentos a essas mulheres, de forma a favorecer recursos socioassistenciais, educacionais e profissionalizantes. Além disso, é possível atuar preventivamente, investindo em programas que promovam um planejamento familiar mais eficaz e efetivo para as famílias que utilizam o sistema público de saúde. Nos dias de hoje, os avanços relativos à anticoncepção, têm viabilizado maior poder de decisão aos casais e à própria mulher, e a possibilidade da escolha sobre o momento de ter um filho, se coloca como um fator decisivo para uma gravidez e maternidade/paternidade saudável.

Espera-se, também, que esses resultados possam ajudar no desenvolvimento de novas pesquisas e deem subsídios a políticas voltadas a gestantes usuárias de serviços públicos. A intenção é proporcionar uma assistência à saúde que ultrapasse o âmbito biomédico e leve em conta as vulnerabilidades emocionais e sociais a partir do desenvolvimento de abordagens que, em última análise, melhorem a qualidade de vida dessa população.

\section{Referências}

Adedinsewo, D. A., Fleming, A. S., Steiner, M., Meaney, M. J., \& Girard, A. W. (2014). Maternal anxiety and breastfeeding: Findings from the MAVAN (Maternal Adversity, Vulnerability and Neurodevelopment) study. Journal Human Lactation, 30(1), 102-109. doi: $10.1177 / 0890334413504244$

Agrati, D., Browne, D., Jonas, W., Meaney, M., Atkinson, L., Steiner, M., \& Fleming, A. S. (2015). Maternal anxiety from pregnancy to 2 years postpartum: Transactional patterns of maternal early adversity and child temperament. Archives of Women's Mental Health, 18(5), 693-705. doi: 10.1007/s00737-014-0491-y

Airosa, S., \& Silva, I. (2013). Associação entre vinculação, ansiedade, depressão, estresse e suporte social na maternidade. Psicologia, Saúde e Doença, 14(1), 64-77. Recuperado em http://www.scielo.mec.pt/pdf/psd/v14n1/ v14n1a05.pdf

Alves, A. M., Gonçalves, C. S. F., Martins, M. A., Silva, S. T., Auwerter, T. C., \& Zagonel, I. P. S.
(2007). A enfermagem e puérperas primigestas: Desvendando o processo de transição ao papel materno. Cogitare Enfermagem, 12(4), 416-427. Recuperado em revistas.ufpr.br/cogitare/article/ download/10063/6918

Alves, J. A. G., Alves, N. G., Ibiapina, F. L. P., Busgaib, R. P. S., \& Costa, F. S. (2010). Ameaça de aborto: Conduta baseada em evidências. Femina, 38(2), 111-115. Recuperado em http:// files.bvs.br/upload/S/0100-7254/2010/v38n2/ a010.pdf

Araújo, D. M. R., Pacheco, A. H. R. N., Pimenta, A. M., \& Kac, G. (2008). Prevalência e fatores associados a sintomas de ansiedade em uma coorte de gestantes atendidas em um centro de saúde do município do Rio de Janeiro. Revista Brasileira de Saúde Materno Infantil, 8(3), 333340. doi: 10.1590/S1519-38292008000300013

Baptista, M. N., Baptista, A. S. D., \& Torres, E. C. R. (2006). Associação entre suporte social, depressão e ansiedade em gestantes. PSIC. Revista de Psicologia da Vetor Editora, 7(1), 39-48. Recuperado em http://pepsic.bvsalud.org/pdf/psic/ v7n1/v7n1a06.pdf

Beltrami, L., Moraes, A. G., \& Souza, A. P. R. A. (2013). Ansiedade materna puerperal e risco para o desenvolvimento infantil. Distúrbios da Comunicação, 25(2), 229-239. Recuperado em http://revistas.pucsp.br/index.php/dic/article/ view/16476/12373

Bener, A. (2013). Psychological distress among mothers of preterm infants and associated factors: A neglected public health problem. Revista Brasileira de Psiquiatria, 35(3), 231-236. doi: 10.1590/1516-4446-2012-0821

Biaggio, A., \& Natalício, L. (2003). Inventário de ansiedade traço-estado - IDATE. Rio de Janeiro, RJ: Centro de Psicologia Aplicada. (Original publicada em 1979)

Borges, A. L. V., Santos, O. A, Nascimento, N. C., Chofakian, C. B. N., \& Gomes-Sponholz, F. A. (2016). Preparo pré-concepcional entre mulheres brasileiras e a relação com o planejamento da gravidez. Revista da Escola de Enfermagem da USP, 50(2), 208-216. doi: 10.1590/S0080623420160000200005

Borges, D. A., Ferreira, F. R., Mariutti, M. G., \& Almeida, D. A. (2011). A depressão na gestação: Uma revisão bibliográfica. Revista de Iniciação Cientifica da Libertas, 1(1), 85-99. Recuperado em http://www.libertas.edu.br/revistalibertas/ revistalibertas1/artigo6.pdf 
Conceição, S. P., \& Fernandes, R. A. Q. (2015). Influência da gravidez não planejada no tempo de aleitamento materno. Escola Anna Nery, 19(4), 600-605. doi: 10.5935/1414-8145.20150080

Camarneiro, A. P. F., \& Justo, J. M. R. M. (2012). Efeito do número de filhos na satisfação conjugal e na vinculação pré-natal materna e paterna. International Journal of Developmental and Educational Psychology, 1(1), 19-28. Recuperado em http://infad.eu/RevistaINFAD/wp-content/ uploads/2013/02/INFAD_010124_19-28.pdf

Caetano, A. J. (2014). Esterilização cirúrgica feminina no Brasil, 2000 a 2006: Aderência à lei de planejamento familiar e demanda frustrada. Revista Brasileira de Estudos de População, 31(2), 309-331. doi: 10.1590/S010230982014000200005

Coelho, H. F., Murray, L., Royal-Lawson, M., \& Cooper, P. J. (2011). Antenatal anxiety disorder as a predictor of postnatal depression: A longitudinal study. Journal of Affective Disorders, 129(1-3), 348-353. doi: 10.1016/j. jad.2010.08.002

Faisal-Cury, A., Araya, R., Zugaib, M., \& Menezes, P. R. (2010). Common mental disorders during pregnancy and adverse obstetric outcomes. Journal of Psychosomatic Obstetrics \& Gynecology, 31(4), 22-235. doi: $10.3109 / 0167482 X .2010 .512404$

Faisal-Cury, A., \& Menezes, P. R. (2006). Ansiedade no puerpério: Prevalência e fatores de risco. Revista Brasileira de Ginecolologia e Obstetrícia, 28(3), 171-178. doi: 10.1590/S010072032006000300006

Fernandes, D. L., Albuquerque, N. L. A., Melo, E. A., Silva, R. B., Vasconcelos, E. M. R., \& Leite, H. P. O. (2012). Percepção de um grupo de gestantes detentoras de história de aborto em gestação anterior. Revista de Atenção à Saúde, 10(32), 47-53. doi: 10.13037/rbcs.vol10n32.1592

Figueiredo, B., \& Conde, A. (2011). Anxiety and depression in women and men from early pregnancy to 3-months postpartum. Archives of Women's Mental Health, 14(3), 247-55. doi: 10.1007/ s00737-011-0217-3

Fonseca-Machado, M. O., Alves, L. C., Freitas, P. S., Monteiro, J. C. S., \& Gomes-Sponholz, F. (2014). Mental health of women who suffer intimate partner violence during pregnancy. Investigación y Educación em Enfermaría, 32(2), 291-305. doi: 10.1590/S0120-53072014000200012
Freire, T. C. G., \& Chatelard, D. S. (2009). O aborto é uma dor narcísica irreparável? Revista Mal Estar e Subjetividade, 9(3), 1007-1022. Recuperado em http://pepsic.bvsalud.org/pdf/malestar/ v9n3/12.pdf

Lopes, R. C. S., Vivian, A. G., Oliveira, D. S., Pereira, C. R. R., \& Piccinini, C. A. (2012). Desafios para a maternidade decorrentes da gestação e do nascimento do segundo filho. In C. A. Piccinini \& P. Alvarenga (Eds.), Maternidade e paternidade: A parentalidade em diferentes contextos (pp. 295-318). São Paulo, SP: Casa do Psicólogo.

Moura, E. R. F., Silva, R. M., Gomes, A. M. A., Almeida, P. C., \& Evangelista, D. R. (2010). Perfil demográfico e de saúde reprodutiva de mulheres atendidas em planejamento familiar no interior do Ceará. Revista Baiana de Saúde Pública, 34(1), 119-133. Recuperado em http:// inseer.ibict.br/rbsp/index.php/rbsp/article/ viewFile/22/22

Morais, M. L. S., Lucci, T. K., \& Otta, E. (2013). Postpartum depression and child development in first year of life. Estudos de Psicologia (Campinas), 30(1), 7-17. doi: 10.1590/S0103-166 X2013000100002

Nardi, C. G. A., Rodrigues, O. M. P. R., Melchiori, L. E., Salgado, M. H., \& Tavano, L. D'. (2015). Bebês com sequência de Pierre Robim: Saúde mental materna e interação mãe-bebê. Estudos de Psicologia (Campinas), 32(1), 129-140. doi: 10.1590/0103-166X2015000100012

O’Donnell, K. J., Glover, V., Barker, E. D., \& O'Connor, T. G. (2014). The persisting effect of maternal mood in pregnancy on childhood psychopathology. Development and Psychopathology, 26(2), 393-403. doi: 10.1017/ S0954579414000029

O’Donnell, K. J., Jensen, A. B., Freeman, L., Khalife, N., O’Connor, T. G., \& Glover, V. (2012). Maternal prenatal anxiety and downregulation of placental 11beta-HSD2. Psychoneuroendocrinology, 37(6), 818-826. doi: 10.1016/j.psyneuen.2011.09.014

Penaforte, M. C. L. F., Silva, L. R., Esteves, A. P. V. S., Silva, R. F., Santos, I. M. M., \& Silva, M. D. B. (2010). Conhecimento, uso e escolha dos métodos contraceptivos por um grupo de mulheres de uma unidade básica de saúde em Teresópolis, RJ. Cogitare Enfermagem, 15(1), 124-130. Recuperado em http://revistas.ufpr.br/cogitare/ article/viewFile/17183/11318 
Pereira, C. R. V. R., Fonseca, V. M., Oliveira, M. I. C., Souza, I. E. O., \& Mello, R. R. (2013). Avaliação de fatores que interferem na amamentação na primeira hora de vida. Revista Brasileira de Epidemiologia, 16(2), 525-534. doi: 10.1590/ S1415-790X2013000200026

Perosa, G. B., Canavez, I. C., Silveira, F. C. P., Padovani, F. H. P., \& Peraçou, J. C. (2009). Sintomas depressivos e ansiosos em mães de recém-nascidos com e sem malformações. Revista Brasileira de Ginecologia e Obstetrícia, 31(9), 433-439. doi: 10.1590/S0100-72032009000900003

Perosa, G. B., Silveira, F. C. P., \& Canavez, I. C. (2008). Ansiedade e depressão de mães de recém-nascidos com malformações visíveis. Psicologia: Teoria e Pesquisa, 24(1), 29-35. doi: 10.1590/S0102-37722008000100004

Pitilin, E. B., Harecemiw, A., Marcon, S. S., \& Pelloso, S. M. (2013). Family support in the everyday life of multiparous women. Revista Gaúcha de Enfermagem, 34(4), 14-20. doi: 10.1590/S198314472013000400002

Prates, C. B., Abib, G. M. C., \& Oliveira, D. L. L. C. (2008). Poder de gênero, pobreza e anticoncepção vivencia de multíparas. Revista Gaúcha de Enfermagem, 29(4), 604-611. Recuperado em http://www.lume.ufrgs.br/bitstream/handle $/ 10183 / 23610 / 000681435 . p d f$ ?sequence $=1$

Prietsch, S. O. M., González-Chica, D. A., Cesar, J. A., \& Mendoza-Sassi, R. A. (2011). Gravidez não planejada no extremo Sul do Brasil: Prevalência e fatores associados. Caderno de Saúde Pública, 27(10), 1906-1916. doi: 10.1590/ S0102-311X2011001000004

Roos, A., Faure, S., Louchner, C., Vythilingum, B., \& Stein, D. J. (2013). Predictors of distress and anxiety during pregnancy. African Journal of Psychiatry, 16(2), 118-122. doi: 10.4314/ajpsy. v16i2.15

Saviani-Zeoti, F., \& Petean, E. B. L. (2015). Apego materno-fetal, ansiedade e depressão em gestantes com gravidez normal e de risco: Estudo comparativo. Estudos de Psicologia (Campinas), 32(4), 675-683. doi: 10.1590/0103$166 \times 2015000400010$
Schetter, C. D., \& Tanner, L. (2012). Anxiety, depression and stress in pregnancy: Implications for mother, children, research, and practice. Current Opinion in Psychiatry, 25(2), 141-148. doi: 10.1097/YCO.0b013e3283503680

Severinski, N. S., Mamula, O., Severinski, S. K., \& Mamula, M. (2009). Maternal and fetal outcomes in grand multiparous women. International Federation of Gynecology and Obstetrics, 107(1), 63-64. doi: 10.1016/j.ijgo.2009.05.011

Simas, F. B., Souza, L. V., \& Scorsolini-Comim, F. (2013). Significados da gravidez e da maternidade: Discursos de primíparas e multíparas. $R e$ vista Psicologia: Teoria e Prática, 15(1), 19-34. Recuperado em http://pepsic.bvsalud.org/pdf/ ptp/v15n1/02.pdf

Spielberger, C. D., Gorsuch, R. L., \& Lushene, R. E. (1970). The State-Trait Anxiety Inventory (Test Manual). Palo Alto, CA: Consulting Psychologists Press.

Veríssimo, S. M. A. C. (2010). Relações entre ansiedade estado e ansiedade traço, sintomas depressivos e sensibilidade ao estresse em puérperas (Dissertação de mestrado) Universidade Lusófona de Humanidades e Tecnologias, Lisboa.

World Health Organization. (1986). Young People's Health - A Challenge for Society. Report of a WHO Study Group on Young People and Health for All (Technical Report Series 731). Geneva: Author.

Ystrom, E (2012) Breastfeeding cessation and symptoms of anxiety and depression: A longitudinal cohort study. BMC Pregnancy Childbirth, 12(36), 1-6. doi: 10.1186/1471-2393-12-36 